\title{
Evolução nutricional de crianças hospitalizadas e sob acompanhamento nutricional ${ }^{1}$
}

\author{
Nutritional evolution of hospitalized children \\ who were under nutritional orientation
}

Ana Flávia de OLIVEIRA²

Fernanda Luísa Ceragioli OLIVEIRA ${ }^{3}$

Yara JULIANO ${ }^{4}$

Fábio ANCONA-LOPEZ ${ }^{3}$

\section{RE S U M O}

\section{Objetivos}

Este estudo teve como finalidade avaliar a evolução nutricional de crianças hospitalizadas com doenças infecciosas, que estiveram sob acompanhamento nutricional.

\section{Métodos}

Foram estudadas 125 crianças com idade de 6 a 36 meses de vida, admitidas na enfermaria de infectologia pediátrica do Hospital São Paulo, durante o período de Março de 2001 a Dezembro de 2002. As avaliações do estado nutricional e da ingestão energética basearam-se nos dados obtidos em inquéritos alimentares e medições antropométricas.

\section{Resultados}

Das crianças avaliadas, 67 eram do sexo masculino (53,6\%); a mediana de idade foi 17 meses e o tempo médio de internação, dez dias. Na época da admissão, $24,8 \%$ das crianças estavam desnutridas enquanto $10,0 \%$ delas apresentavam sobrepeso e obesidade. Prevaleceu o diagnóstico de doenças agudas (69,6\%). Realizaram terapia nutricional $21,6 \%$ das crianças, sendo que destas, $81,5 \%$ receberam terapia nutricional via oral. 0 grupo de desnutridos teve melhora significativa do escore- $Z$ de peso para estatura- $Z$ peso/estatura $(p=0,001)$; o grupo de eutróficos não teve alteração significativa de escore-Z peso/estatura $(p=0,651)$ e o grupo com sobrepeso/obesos teve redução significativa do escore-Z peso/estatura $(p=0,026)$. Não houve associação significativa entre realização de terapia nutricional e melhora do estado nutricional $(p=0,37)$. A melhora do

\footnotetext{
1 Artigo elaborado a partir da dissertação de A.F. OLIVEIRA, "Evolução da condição nutricional de crianças de 6 a 36 meses internadas em enfermaria de infectologia pediátrica". Universidade Federal de São Paulo, 2004.

2 Programa de Pós-graduação em Nutrição, Departamento de Pediatria, Universidade Federal de São Paulo, Escola Paulista de Medicina. Rua Loefgreen, 1647, Vila Clementino, 04040-032, São Paulo, SP, Brasil. Correspondência para/Correspondence to: A.F. OLIVEIRA. E-mail: <anaflavia@fag.edu.br>.

3 Disciplina de Nutrição e Metabolismo, Departamento de Pediatria, Universidade Federal de São Paulo, Escola Paulista de Medicina. São Paulo, SP, Brasil.

4 Disciplina Saúde Coletiva, Faculdade de Medicina, Universidade de Santo Amaro, São Paulo, SP, Brasil.
} 
escore-Z peso/estatura esteve relacionada à ingestão de energia maior que a recomendação normal para a idade $(p<0,001)$.

\section{Conclusão}

As crianças acompanhadas neste estudo tiveram melhora significativa do estado nutricional, tornando-se evidente a importância do acompanhamento nutricional durante a internação.

Termos de indexação: criança, dietoterapia, estado nutricional, hospitalização.

\section{A B S T R A C T}

\section{Objective}

This study aimed at evaluating the nutritional evolution of hospitalized children with infectious diseases, who were put under nutritional orientation.

\section{Methods}

Evaluate the effect of therapeutic nutritional orientation on 125 children between 6 and 36 months of age, who were admitted at the Pediatric Infectology Ward of the Hospital São Paulo, São Paulo, Brazil, between March 2001 and December 2002. The evaluations of nutritional status and energetic intake were based on data obtained from inquiry-forms on feeding-patterns and anthropometric measurements.

\section{Results}

Of the children being evaluated, 53,6\% (67) were male. The mean age was 17 months, and the average period spent in the hospital was 10 days. At admission, 24,8\% (31) of the children were undernourished and 10,0\% (23) were overweight or obese. The diagnosis of acute diseases affected 69,6\% (87) of the children. Nutritional support was provided for $21.6 \%$ (27) of the children, out of which, $81.5 \%$ (22) received nutritional support orally. The undernourished group presented a significant improvement of the Z-score of Weight over Height - Z WIH ( $\mathrm{p}=0.001)$; the eutrophic group had no significant change of the Z-score Weight over Height $(\mathrm{p}=0.651)$, and the group overweight/obese showed a significant reduction of the Z-score Weight over Height $(p=0.026)$. No significant associations were found between nutritional support therapy and improvement of the nutritional status $(\mathrm{p}=0.37)$. The children who had an energy intake higher than the one recommended for their age, had a significant improvement of the Z-score Weight over Height $(\mathrm{p}<0.001)$.

\section{Conclusion}

The children followed up in this study had their nutritional condition significantly improved, making it evident that nutritional orientation, or diet therapy, can improve the treatment of hospitalized children.

Indexing terms: child, dietotherapy, nutritional state, hospitalization.

\section{N T R O D U Ç Ã O}

A interação sinérgica entre desnutrição e infecção é reconhecida há muito tempo com base em observações clínicas e em dados epidemiológicos. Como resultado final ocorre a potencialização de cada uma isoladamente e adicionadas entre si, sendo que a desnutrição compromete as defesas imunológicas do hospedeiro, facilitando a instalação de processos infecciosos e, por outro lado, as infecções reiteradas comprometem o estado nutricional, tornando-se um círculo vicioso ${ }^{1,2}$.
Alterações na relação nutrição e imunidade decorrente de processo infeccioso podem favorecer a desnutrição por meio de infecções intestinais capazes de alterar a absorção e a biodisponibilidade de nutrientes, de processo febril que acarreta aumento do requerimento energético e de infecções crônicas que aumentam a glicogênese e a lipogênese, alterando o metabolismo de carboidratos, lipídeos, proteínas, níveis de micronutrientes e balanço eletrolítico, além de acarretar alterações hormonais que interferem no metabolismo de nutrientes ${ }^{3-5}$. 
Em geral, a resposta metabólica do hospedeiro durante a infecção independe da sua etiologia. No entanto, a intensidade dessa resposta relaciona-se com a virulência do agente infeccioso e o estado de imunocompetência do hospedeiro, condição nutricional, fatores genéticos e presença eventual de outras doenças associadas. Dependendo da gravidade da infecção e da resposta imune do hospedeiro, as alterações metabólicas decorrentes podem predispor à desnutrição aguda, à síndrome da disfunção múltipla de órgãos e à morte 1 .

A nutrição tem sido, ao longo do tempo, negligenciada pela medicina terapêutica e preventiva por falta de reconhecimento dos profissionais de saúde da sua importância no processo saúde-doença; além disso, soma-se à dificuldade de reconhecimento do paciente com distúrbios nutricionais a dificuldade de estabelecer associações desses com as várias enfermidades ${ }^{5-7}$.

No hospital, a desnutrição, muitas vezes, é pouco reconhecida e nem sempre é tratada, com conseqüente aumento da morbidade e mortalidade, principalmente por infecções. O reconhecimento precoce e o tratamento eficaz diminuiriam o tempo de hospitalização, minimizariam as ações hospitalares nutricionalmente iatrogênicas e transtornos familiares ${ }^{6,8}$.

A terapêutica nutricional, dependendo da doença de base, é bastante variável ${ }^{9}$. Por isso, a avaliação nutricional, além de ser realizada quando da admissão do paciente, deve ser continuada durante o período da internação para controle mais racional do tratamento e recuperação do estado normal de saúde e nutrição ${ }^{6,8}$.

Demonstrada claramente a relação nutrição, imunidade e infecção, é de fundamental importância que se considere, na abordagem dos indivíduos com doenças infecciosas, o acompanhamento do estado nutricional para que haja redução da suscetibilidade e aumento da resistência à infecção $0^{3,5}$.

Foi propósito deste estudo avaliar a evolução nutricional de crianças hospitalizadas em enfermaria de infectologia pediátrica sob acompanhamento nutricional.

\section{MÉ T O D O S}

Trata-se de ensaio terapêutico sem grupo controle que avaliou o estado nutricional, o tipo de doença, a ingestão energética durante a doença e o tipo de terapia nutricional realizada. Fizeram parte deste estudo 125 crianças admitidas na enfermaria de infectologia pediátrica do Hospital São Paulo, com idade entre 6 e 36 meses de vida, de março de 2001 a dezembro de 2002. Crianças internadas no final de semana e que tiveram alta na segunda-feira e aquelas nas quais não foi possível realizar medidas antropométricas durante a internação foram excluídas do estudo.

No período máximo de 48 horas após a internação, realizou-se avaliação clínico-nutricional dos pacientes admitidos, coletando dados do prontuário e complementando-os, quando necessário, com informações obtidas da mãe ou responsável. A avaliação nutricional foi realizada por inquéritos alimentares e medidas antropométricas. Para o controle da ingestão energética, foi realizado, durante a internação, registro de 24 horas da ingestão alimentar duas vezes na semana.

Para analisar a doença de base e devido à grande variedade de doenças como causa de internação, optou-se por dividi-las em dois grandes grupos: doenças agudas e crônicas. Foram classificadas em doenças agudas: doenças de pele (escabiose, celulites, dermatites, abscessos infecciosos, varicela infectada etc), infecções de vias aéreas superiores e inferiores, infecções do trato urinário, diarréias infecciosas, artrites e pioartrites e meningites. E em doenças crônicas: neuropatias, síndrome da imune deficiência adquirida (SIDA), tuberculoses, cardiopatias congênitas, osteomielite e fibrose cística. Pacientes portadores de doenças crônicas agudizadas foram incluídos no grupo de doenças crônicas. 
Para a classificação do estado nutricional foram utilizados os escores- $Z$ de peso para estatura (Z P/E) e estatura para idade (Z E/I). O cálculo desses índices foi efetuado com base no padrão National Center for Health Statistics (NCHS) de 1979. O critério de classificação adotado para caracterização do estado nutricional foi o proposto pela Organização Mundial da Saúde adaptado, que utiliza o escore-Z de peso para estatura e estatura para idade: desnutrição grave: escore-Z <-3 para o índice P/E; desnutrição moderada: escore-Z >-3 e $\leq-2$ para o índice P/E; desnutrição leve: escore-Z $>-2$ e $\leq-1$ para o índice $P / E$; eutrofia: escore-Z $>-1$ e $<1$ para o índice $P / E$; sobrepeso: escore $Z \geq 1$ e $<2$ para o índice P/E; obesidade: escore- $Z \geq 2$ para o índice P/E; baixa estatura: escore-Z $\geq-2$ para o índice E/l; risco de baixa estatura: escore-Z $>-2$ e $\leq-1$ para o índice E/l; sem comprometimento estatural: escore-Z $>-1$ para o índice E/l.

Para efeitos de análise estatística, formaram-se três grupos: desnutridos ( $\geq-1$ escore-Z), eutróficos (>-1 e < 1 escore-Z) e com sobrepeso/ obesos (> 1 escore-Z).

A conduta nutricional variou de acordo com o estado nutricional e clínico do paciente. Os cálculos de recomendação para macro e micronutrientes foram feitos de acordo com a Recommended Dietary Allowances ${ }^{10}$. A padronização da conduta nutricional para os desnutridos crônicos seguiu a recomendação da World Health Organization ${ }^{11}$. Para os desnutridos agudos, eutróficos, com sobrepeso e obesos, o objetivo foi ofertar macro e micronutrientes com o objetivo de adequação ou manutenção do peso. As condutas seguiram o seguinte protocolo:

a) Desnutridos crônicos: suplementação de polivitamínico (complexo $B$, vitaminas $A$ e $C$ ): 1,5 vez maior que a recomendação; suplementação de zinco: $2 \mathrm{mg} / \mathrm{kg} / \mathrm{dia}$; suplementação de cobre: 0,2mg/kg/dia; suplementação ácido fólico: $5 \mathrm{mg}$ na dose inicial e $1 \mathrm{mg}$ por dia após primeiro dia; energia: 1,5 a 2,0 vezes a recomendação por quilo de peso atual.

b) Desnutridos leve/moderado: suplementação de polivitamínico (complexo B, vitaminas A e C): uma vez a recomendação; suplementação de zinco: uma vez a recomendação; energia: 1,0 a 1,5 vezes maior que a recomendação por quilo de peso atual.

c) Eutróficos/sobrepeso/obesos: suplementação de micronutrientes que não são atingidos pela ingestão durante a internação; energia: uma vez a recomendação por quilo de peso atual.

d) Exceções: o cobre não foi ofertado durante a vigência de processo agudo e disfunção hepática; durante o período de infecção aguda, foi ofertada apenas a taxa metabólica basal de energia.

e) Aumento da oferta calórica: com o objetivo de aumentar a ingestão calórica para atingir a recomendação proposta, foram utilizados módulos de carboidratos, lipídeos, proteínas e/ou suplementos completos de acordo com os cálculos da ingestão realizados durante a internação e/ou estado clínico. Os módulos/suplementos utilizados foram: módulos de carboidratos - cereais ou polímeros de glicose (nos casos clínicos de má absorção); módulos de lipídios - triglicerídeos de cadeia longa ou triglicerídeos de cadeia média (nos casos de má absorção); módulos de proteínas - proteína intacta do leite (pouco utilizada); suplementos completos - líquidos embalados em "tetrapack" com carboidratos, proteínas e lipídeos em proporções adequadas, utilizados em sua grande maioria por via oral.

f) Indicação de terapia nutricional: terapia nutricional via oral, quando a ingestão da dieta hospitalar se encontrou abaixo de $60 \%$ da recomendação; terapia nutricional enteral (nasogástrica, pós-pilórica ou ostomias), quando a ingestão oral se encontrou abaixo de $60 \%$ da recomendação após introdução de terapia nutricional via oral ou por impossibilidade de deglutição; terapia nutricional parenteral, quando houve impossibilidade de se utilizar o trato gastrintestinal (não funcionante) ou quando não se atingiram as recomendações mínimas via enteral. 
g) Indicação de fórmulas especiais: foram utilizadas de acordo com a condição clínica e nutricional do paciente (hidrolisada, semi-hidrolisada, isenta de lactose etc.).

h) Leites infantis adaptados para o primeiro ano de vida: na impossibilidade de aleitamento materno, foi padronizada a utilização de fórmulas infantis modificadas segundo a faixa etária no primeiro ano de vida.

Para elaboração do banco de dados foi utilizado o programa Epi Info 6.0. Os questionários foram pré-codificados e checados quanto à sua consistência interna antes de serem liberados para digitação. Estiveram envolvidos nessa tarefa os profissionais da equipe devidamente treinados. Os dados foram transcritos no banco de dados com dupla digitação e posterior validação, visando à correção de erros.

A análise estatística utilizada foi a não paramétrica ${ }^{12}$. Para análise dos resultados foram aplicados os seguintes testes: teste de Wilcoxon para comparar os valores do Escore-Z de Peso para Estatura (Z P/E) nos tempos de admissão e alta hospitalar para desnutridos, eutróficos e com sobrepeso/obesos separadamente; análise de variância de Kruskal-Wallis ${ }^{12}$ com a finalidade de comparar os valores do $\Delta \%$ calculado $(\Delta \%=Z$ P/E depois - Z P/E antes / Z P/E antes $x$ 100) dos grupos de desnutridos, de eutróficos e com sobrepeso; teste de Mann-Whitney ${ }^{12}$ para comparar os dias de internação do grupo de desnutridos e não-desnutridos; teste do qui-quadrado ${ }^{12}$ para possíveis associações entre as variáveis estudadas; fixou-se em $0,05 \%$ ou $5,0 \%(\alpha \leq 0,05)$ o nível de rejeição da hipótese de nulidade.

\section{RES U LT A D O S}

Do total de crianças acompanhadas, $53,6 \%$ (67) eram do sexo masculino. A mediana de idade foi 17 meses (valor mínimo: 6 meses e valor máximo: 36 meses) e tempo de internação de 10 dias (valor mínimo: 3 dias e valor máximo:
120 dias). Quanto ao estado nutricional à admissão, 24,8\% (31) estavam desnutridas (21 levemente, 8 moderadamente e 2 gravemente) e $10,0 \%$ (23) apresentaram sobrepeso e obesidade (13 com sobrepeso e 9 obesos).

A causa de internação por doenças agudas foi prevalente em $69,6 \%$ (87) das crianças, sendo que os diagnósticos encontrados foram: $25,9 \%$ com broncopneumonias/pneumonias, 24,1\% com doenças infecciosas de pele (celulites, dermatites, impetigo e erisipela) e 15,5\% com meningite/ mastoidite. Das crianças internadas por doenças crônicas, as neuropatias foram as mais prevalentes $(31,3 \%)$, seguidas de SIDA com complicações agudas $(21,9 \%)$ e tuberculose $(15,6 \%)$.

Receberam terapia nutricional 21,6\% (27) das crianças, sendo que dessas, 81,5\% (22) receberam terapia nutricional via oral. Apenas quatro crianças receberam nutrição enteral e uma recebeu parenteral.

Para avaliar a evolução nutricional das crianças, foram criados três grupos: desnutridos, eutróficos e com sobrepeso/obesos (Tabela 1). 0 grupo de desnutridos teve melhora significativa do Z P/E $(p=0,001)$, o grupo de crianças eutróficas não teve alteração significativa do Z P/E $(p=0,651)$, e o grupo de crianças com sobrepeso/obesas teve diminuição significativa do Z P/E $(p=0,026)$.

As crianças desnutridas foram as que mais realizaram terapia nutricional (Tabela $2, p=0,001$ ), mas não houve significância estatística entre realização de terapia nutricional e melhora do estado nutricional (Tabela 3, $p=0,37$ ). A melhora do estado nutricional esteve intimamente relacionada com a ingestão energética durante a internação: as crianças que ingeriram mais que uma vez a recomendação de energia por quilo de peso para idade durante a internação foram as que tiveram melhor evolução nutricional (Tabela $4, p<0,001)$. As crianças desnutridas permaneceram mais tempo internadas do que as não desnutridas (Tabela $5, p=0,024$ ). 
Tabela 1. Avaliação da evolução nutricional dos grupos de desnutridos, de eutróficos e com sobrepeso/obesos segundo o Escore-Z de Peso para Estatura (Z P/E).

\begin{tabular}{|c|c|c|c|c|c|c|}
\hline & \multicolumn{2}{|c|}{ Desnutridos } & \multicolumn{2}{|c|}{ Eutróficos } & \multicolumn{2}{|c|}{ Sobrepeso/Obesos } \\
\hline & Admissão & Alta & Admissão & Alta & Admissão & Alta \\
\hline Número de casos & 31,00 & 27,00 & 72,00 & 76,00 & 22,00 & 22,00 \\
\hline Média Z P/E & $-1,80$ & $-1,56$ & 0,17 & 0,21 & 1,96 & 1,64 \\
\hline DP & 0,74 & 0,72 & 0,57 & 0,64 & 0,91 & 0,86 \\
\hline
\end{tabular}

Teste do Wilcoxon= Desnutridos: $p=0,001$ ( $z$ calculado: 3,26 z crítico: 1,96); Eutróficos: $p=0,651$ (z calculado: 0,45 z crítico: 1,96); Sobrepeso/ Obesos: $p=0,026$ ( $z$ calculado: 2,21 z crítico: 1,96); Análise de Variância de Kruskal-Wallis: $\Delta \%$ desnutridos $\chi \Delta \%$ eutróficos $\chi \Delta \%$ sobrepeso/ obesos: $p<0,001$, onde desnutridos $>$ eutróficos $>$ sobrepeso/obeso $\chi^{2}$ calculado $=22,77 \chi^{2}$ crítico $=3,84$ ).

Tabela 2. Associação entre estado nutricional quando da admissão e realização de terapia nutricional.

\begin{tabular}{lcccc}
\hline Estado nutricional & Com TN & Sem TN & Total & $\%$ TN \\
\hline Desnutrido & 14 & 17 & 31 & 45,2 \\
Eutrófico & 10 & 61 & 71 & 14,1 \\
Sobrepeso/Obesos & 3 & 20 & 23 & 13,0 \\
\hline Total & 27 & 98 & 125 & 21,6 \\
\hline
\end{tabular}

* Não receberam porque atingiam as necessidades pela alimentação normal; Teste do qui-quadrado: $p=0,001$ ( $\chi^{2}$ calculado=5,99 $\chi^{2}$ crítico=3,84); $\mathrm{TN}=$ terapia nutricional.

Tabela 3. Associação entre realização de terapia nutricional e melhora do estado nutricional.

\begin{tabular}{lcccc}
\hline Estado nutricional & Melhorou/inalterado & Piorou & Total & \% de melhora/inalterado \\
\hline Recebeu & 18 & 9 & 27 & 66,7 \\
Não Recebeu & 42 & 56 & 98 & 57,1 \\
\hline Total & 74 & 51 & 125 & 59,2 \\
\hline
\end{tabular}

Teste do qui-quadrado: $p=0,37\left(\chi^{2}\right.$ calculado $=0,80 \chi^{2} \quad$ crítico $\left.=3,84\right)$.

Tabela 4. Associação entre ingestão energética e evolução nutricional.

\begin{tabular}{lcccc}
\hline $\begin{array}{l}\text { Ingestão } \\
\text { energética }\end{array}$ & $\begin{array}{c}\text { Melhorou/ } \\
\text { inalterado }\end{array}$ & Piorou & Total & $\begin{array}{c}\text { \% de melhora/ } \\
\text { inalterado }\end{array}$ \\
\hline$\leq 1$ RDA & 24 & 27 & 51 & 47,1 \\
$>1$ RDA & 26 & 5 & 31 & 83,9 \\
\hline Total & 50 & 32 & 82 & 61,0 \\
\hline
\end{tabular}

Teste do qui-quadrado: $p<0,001$ ( $\chi^{2}$ calculado=10,98 $\chi^{2}$ crítico=3,84)

\section{I S C U S S Ã O}

O principal objetivo do acompanhamento nutricional em pacientes hospitalizados é realizar
Tabela 5. Associação entre tempo de internação e estado nutricional.

\begin{tabular}{lcc}
\hline Dias & Desnutridos & Não desnutridos \\
\hline Mediana & 12,0 & 8,5 \\
Média & 14,8 & 12,4 \\
DP & 8,9 & 14,7 \\
\hline
\end{tabular}

Teste de Mann-Whitney: $p=0,024$ (z calculado: $-2,26$ z crítico: 1,96).

terapia nutricional adequada à doença e ao estado nutricional apresentado, colaborando assim com a melhora do prognóstico do paciente $\mathrm{e}^{6,8,9,13-15}$. Logo, o objetivo não foi realizar recuperação do 
estado nutricional das crianças internadas, mas a manutenção do peso corporal, evitando que os pacientes sofram depleção durante a internação. Portanto, consideraram-se como tendo tido evolução nutricional satisfatória as crianças que não perderam peso.

As crianças desnutridas tiveram melhora significativa do Z P/E durante a internação. Alguns fatores podem explicar o ocorrido, como a maior permanência de tempo de internação ${ }^{15-21}$, fazendo com que recebessem, portanto, maior tempo de acompanhamento nutricional, e o fato de o término da fase aguda da doença ocorrer durante a internação, com diminuição dos sintomas clínicos de infecção (febre, anorexia, sonolência) devido à diminuição das Interleucinas 1 e 6, acarretando aumento da ingestão alimentar ainda no período da hospitalização 1,4,5,22-24. A maior carência nutricional aliada à avidez orgânica, a facilidade de acesso aos alimentos concomitantemente à monitoração da ingestão alimentar e a qualidade adequada de nutrientes oferecidos contribuem para melhora do estado nutricional.

As crianças consideradas eutróficas mantiveram o Z P/E, o que se considerou evolução satisfatória, pois não houve deterioração do estado nutricional durante a internação. As crianças que entraram com diagnóstico nutricional de sobrepeso ou obesidade tiveram redução significativa do Z P/E; apesar de esse não ser o melhor momento para a perda de peso, o fato pode ser explicado por restrição dos alimentos hipercalóricos (salgadinhos, refrigerantes, doces) e sua substituição por alimentação adequada ofertada durante a internação. Apesar de não existir controle rígido da entrada de alimentos de fora do hospital, a orientação dada aos pacientes consiste na proibição desse comportamento.

Pacientes desnutridos são os maiores candidatos a terapia nutricional ${ }^{20-22,25}(p=0,001)$ por terem maior necessidade, porém o recebimento de terapia nutricional não teve associação significativa com a melhora do estado nutricional, mas com a ingestão energética durante a internação. Os pacientes que tiveram ingestão energética média acima da recomendação por quilo de peso para idade durante a internação tiveram associação significativa com a melhora do estado nutricional $(p<0,001)$ porque, nessa fase, o organismo em vigência de infecção necessita de maior requerimento energético devido à febre e às alterações no metabolismo de macro e micronutrientes aliadas ao seqüestro de aminoácidos para a síntese de proteínas de fase aguda ${ }^{3,4,23-25}$. Assim, com a adaptação da dieta hospitalar aos hábitos alimentares e necessidades da criança, aquelas que ingeriram mais que as recomendações de energia para a idade tiveram melhor evolução nutriciona|21,26,27.

Esses fatos evidenciam que o acompanhamento nutricional contribui para manutenção e/ou melhora do estado nutricional do paciente durante a hospitalização.

\section{O N CLUS Ã O}

As crianças acompanhadas neste estudo tiveram manutenção ou melhora significativa do estado nutricional, evidenciando a necessidade do acompanhamento nutricional durante a hospitalização. Ressalta-se a importância da atuação em equipe multiprofissional para que haja melhor tratamento da criança hospitalizada.

\section{A G RADECIMENTOS}

Ao Dr. Antônio Vladir Lazzetti e a toda equipe médica da enfermaria. Às nutricionistas Laura, Érica, Flávia, Juliana, Rosângela, Fernanda, Mile, Mariana, Ana Flávia e Marina, pelo auxílio na coleta e codificação dos dados.

\section{REFERÊ NCIAS}

1. Farhat $\mathrm{CL}$, Faria $\mathrm{SM}$. Infecciosas. Distúrbios da Nutrição. 1998; 6(33):203-9. 
2. Corish CA, Kennedy NP. Protein-energy undernutrition in hospital in-patients. Brit J Nutr. 2000; 83(6):575-91.

3. Meira DA. Interação Infecção, nutrição e imunidade. Rev Soc Bras Med Trop. 1995; 28(4):315-9.

4. Jardim RF, et al. Citocinas. Rev Bras Alerg Imunol. 17(3): 86-97; 1994

5. Chandra RK. Nutrition and immunology: from the clinic to cellular biology and back again. Proc Nutr Soc. 1999; 58(3):681-3.

6. Valente FLS, Corso ACT. Implantação de Sistema de Vigilância Alimentar e Nutricional junto aos pacientes internados no Hospital Universitário da Universidade Federal de Santa Catarina. Rev Cien Saúde. 1992; 6(1):48-68.

7. Leleiko NS, Luder E, Fridman M, Fersel J, Benkov K. Nutritional assessment of pediatric patients admitted to in acute-care pediatric service utilizing anthropometric measurements. JPEN. 1986; 10(2):166-8.

8. Batista Filho M, Costa MJCC. Desnutrição Energético-Protéica: A experiência do Hospital Universitário de João Pessoa, PB. III - Evolução dos Casos. Revista do IMIP. 1990; 4(1):15-8.

9. Romaldini CC, Margarida NT, Bueno L, Tanikawa CE, Cardoso AL, Carraza FR. Avaliação do estado nutricional de crianças hospitalizadas. Rev Paul Ped. 1996; 14(4):149-52.

10. National Research Council. Committee on Diet and Health. Food and Nutrition Board. Diet and health: implication for reducing chronic disease risk. Washington (DC): National Academy Sciences. 1989.

11. World Health Organization. Management of severe malnutrition: a manual for physicians and other senior health workers. Geneva; 1999.

12. Siegel S, Castellan Jr NJ. Nonparametric Statistics. 2nd ed. New York: McGraw-Hill; 1988. p.399.

13. Péret Filho LA. Manual de Suporte Nutricional em Gastroenterologia Pediátrica. Belo Horizonte: Medsi; 1994. p.35.

14. Perez JIU, Cesar MJP, Benavent EG, Alvarez-Estrada AM. Deteccion precoz y control de la desnutrición hospitalaria. Nutr Hosp. 2002; 17(3):139-46.

15. Ozturk Y, Buyukgebiz B, Arslan N, Ellidokus H. Effect of hospital stay on nutritional anthropometric data in Turkish children. J Trop Pediatr. 2003; 49(3):189-90.
16. Nóbrega FJ, Queiroz SS, Trindade CEP. Repercussões da desnutrição energético-protéica. Distúrbios da Nutrição. 1998; 6(32):188-95.

17. Murayama AG, Fujimori MM, Leite WA. Morbidade e estado nutricional de crianças hospitalizadas. Ped Atual. 1995; 8(4):65-72.

18. Pizarro T, Rodriguez L, Atalah E. Diagnóstico de ingreso y evolución nutricional beneficiaries. Rev Med Chile. 2003; 131(9):1031-6.

19. Watanabe S, Cukier C, Magnoni D, Guimarães RN, Urenhiuki KL, Rauba A. Nutrição enteral precoce reduz tempo de internação hospitalar e melhora reembolso diário do Sistema Único de Saúde ao hospital. Rev Bras Nutr Clin. 2002; 17(2): 47-50.

20. Merhi VLA, Garcia RWD, Tafner B, Calheiros FM, Casteli R, Aquino JLB. Relação entre estado nutricional e as características clínicas de pacientes internados em enfermaria de cirurgia. Rev Ciên Méd. 2000; 9(3):105-14.

21. Stanga Z, Zurfluh $Y$, Roselli M, Sterchi AB, Tanner $B$, Knecht G. Hospital food: a survey of patients' perceptions. Clin Nutr. 2003; 22(3):241-6.

22. Leão RC, Forte WCN, Campos JVM. A imunidade inespecífica em crianças com má nutrição calórico-protéica moderada. Rev Bras Alerg Imunol. 1985; 8(1):5-8.

23. Menezes $H$, Bertola E. A inter-relação entre nutrição e imunidade. Nutrição em Pauta. 2001; 49:36-40.

24. Bhaskaram P. Nutritional modulation of immunity to infection. Indian J Pathol Microbiol. 1992; 35(4):392-400.

25. Moreira FLM, Padovani CR, Maffei HVL. Evolução antropométrica de crianças hospitalizadas com diarréia persistente e desnutrição grave, submetidas a suporte nutricional. J Ped. 1996; 72(4):235-41.

26. Dupertuis YM, Kossovsky MP, Kyle UG, Raguso CA, Genton L, Pichard C. Food intake in 1707 hospitalized patients: a prospective comprehensive hospital survey. Clin Nutr. 2003; 22(2):115-23.

27. Kondrup J, Johansen N, Plum LM, Bak L, Larsen IH, Martinsen $A$, et al. Incidence of nutritional risk and causes of inadequate nutritional care in hospitals. Clin Nutr. 2002; 21(6):4461-8.

Recebido para publicação em 12 de setembro de 2003 e aceito em 3 de setembro de 2004. 